\title{
DETERMINATION OF THE DECREE OF HUMIFICATION IN PEAT SAMPLES
}

\author{
By \\ Armi KaIla \\ University of Helsinki, Department of Agricultural Chemistry \\ Received December 3, 1955
}

The degree of humification is a valuable characteristic of peat samples, important not only for soil chemists but also for agricultural and industrial purposes. Yet, it seems to be a quantity the estimation of which is not quite simple. One of the main reasons for this situation probably lies in the fact that we have no very clear conception of the exact meaning of the degree of humification: we do not know what we try to determine.

The general trends in the decomposition of organic material are rather well known. The content of typical plant carbohydrates decreases more or less rapidly; proteins, polyuronides, lignin like substances and humic acids tend to accumulate. Simultaneously the decomposing organic material loses its cell structure and turns dark brown. This holds true also with peat formation, although the conditions under which it takes place often markedly retard and modify the processes. For example, under anaerobic and acid conditions common in peat deposits the synthesis of true humic acids can be largely prevented.

It is obvious that the degree of humification is a relative quantity which could be determined, at least approximately, using any of the chemical or physical characteristics which continually change with an advance of the decomposition. For the sake of comparison the corresponding figures for the original material must be known. In order to get reliable results the other end of the humification scale, the quality of the end product obtainable under the conditions in question, must also be taken into consideration. Often the loss of dry matter during decomposition is a necessary figure. Unfortunately, all these are factors generally not available when the degree of humification of a certain sample is to be estimated. Therefore, the determination must be based on approximate values and the results obtained, by any method, are more or less vague. 


\section{Conventional methods}

In the practical field work in Finland the method adopted by von Post (9) is most commonly used for the determination of the degree of humification. It is based on the direct examination of fresh samples. The colour and turbidity of the water pressed by hand from the sample, and the structure and consistence of the peat residue are the main factors observed. A grading from $\mathrm{H}_{1}$ to $\mathrm{H}_{10}$ is used, in which $\mathrm{H}_{1}$ represents the undecomposed peat. Apparently this method can give satisfactory results provided that the observer is well trained. It is, of course, exposed to subjective errors and, as e.g. Mitchell (8) emphasizes, it can be used only if the samples have retained their full content of water. Often it would be desirable to get a more exact estimation of the degree of humification than can be obtained by this method, and in the ordinary laboratory work other ways must be found for the analyses of dry and ground peat samples.

Among the several procedures proposed for the determination of the degree of humification only a few seem to yield fairly reliable results. Such a method based on the physical characteristics of peat samples is e.g. SEGEBERG's (10) determination of the "Vertorfungszahl», the increase in the volume weight of ash-free dry matter expressed as a percentage of the corresponding weight of volume for the sample to be analyzed. According to SEGEBERG's investigation, also the maximum water holding capacity of a peat sample gives a fairly well picture of the degree of humification. The hygroscopicity, on the other hand, does not characterize the state of humification $(1,3)$.

As one of the most obvious phenomenon during the decomposition of organic matter is its turning dark brown, it is easy to undestand that the intensity of the colour of peat extracts is supposed to correlate with the degree of humification. BEAM in 1912 (ref. 7) appears to be the first soil chemist who has used this principle, but only for the determination of humus in mineral soils. It was Melin and OdéN (7) who on the basis of their studies developed a quantitative colorimetric method for the determination of humification and introduced the "Humifizierungszahl", a relative value obtained by the comparison of the colour intensity of the extract of the unknown sample and of that of the preparation Acidum huminicum by Merck. Sodium hydroxide solution was used for the extraction which was performed at the boiling temperature. This "Humifizierungszahl» has been largely employed by the German soil scientists, in particular, and its determination belongs to the common procedures in the investigation of soil organic matter and peat. Yet, its value has been critized by several authors. Hock (2) considers the use of a standard preparation erroneous, especially because the Acidum huminicum by Merck represents humic acids only from highmoor peat and therefore cannot be suitable for analyzing of other kinds of humic acids. SPRInger (11) emphasizes the fact that the "Humifizierungszahl» does not correlate with the content of true humus or organic matter insoluble in acetyl bromide.

SPRINGER (11) claims that the most reliable way to find out to which stage the decomposition of organic matter has advanced is to determine its content of true 
Table 1. The degree of decomposition expressed by various characteristics

\begin{tabular}{|c|c|c|c|c|c|c|c|c|}
\hline \multirow{2}{*}{ Sample } & \multirow[b]{2}{*}{ v. Post } & \multirow{2}{*}{$\begin{array}{c}\text { Water- } \\
\text { capacity } \\
\%\end{array}$} & \multirow{2}{*}{$\begin{array}{c}\text { V.Z. } \\
\text { Segeberg }\end{array}$} & \multicolumn{3}{|c|}{ Waksman fractions $\%$} & \multirow{2}{*}{$\begin{array}{c}\text { V.G. } \\
\text { Keppeler }\end{array}$} & \multirow{2}{*}{$\begin{array}{l}\text { Colour } \\
\text { number }\end{array}$} \\
\hline & & & & Cellulose & $\begin{array}{l}\text { Hemicel- } \\
\text { lulose }\end{array}$ & Insoluble & & \\
\hline 1. $\mathrm{Sp}$ & 1 & 880 & 25 & 17.6 & 14.5 & 31.3 & 23 & .13 \\
\hline 2. $\mathrm{BC}_{\mathrm{p}}$ & $2-3$ & 650 & 63 & 13.8 & 13.3 & 38.6 & 36 & .11 \\
\hline 3. EuSCp & 3 & 670 & 62 & 11.4 & 12.6 & 41.2 & 31 & .05 \\
\hline 4. CSp & 3 & 380 & 72 & 9.4 & 12.9 & 46.0 & 40 & .31 \\
\hline 5. $\mathrm{LC}_{\mathrm{p}}$ & $3-4$ & 370 & 76 & 7.3 & 10.1 & 41.8 & 33 & .15 \\
\hline 6. $\mathrm{SC}_{\mathrm{P}}$ & 4 & 500 & 71 & 6.5 & 11.8 & 45.7 & 39 & .20 \\
\hline 7. $\mathrm{CSp}$ & 4 & 450 & 73 & 6.3 & 10.3 & 42.9 & 40 & .26 \\
\hline 8. LCSp & 4 & 380 & 77 & 4.6 & 9.2 & 46.6 & 46 & .22 \\
\hline
\end{tabular}

humus. Springer's "Zersetzungsgrad" denotes the ratio of acetyl bromide insoluble carbon to total organic carbon expressed as a percentage, or in samples which are poor in ash, the ratio of insoluble organic material to the loss on ignition. This method is tedious and expensive and the possibility is not excluded that the brom-acetolyse renders real humic acids soluble (6). It also seems to be less suitable for the determination of the degree of humification in peat soils. SPRINGER (11) himself noticed that particularly in the deeper layers of peat the formation of true humic acids is retarded and the apparently rather well humified material contains mostly somewhat changed lignin and other pre-stages of proper humic acids. He also states that it sometimes can be possible to find in the deeper peat layers a decrease in the content of acetyl bromide insoluble matter, due to the decomposition of humic acids. Hence the conclusion must be drawn that the "Zersetzungsgrad" is not a recommendable quantity for the determination of the degree of humification in peat samples, although it can offer other valuable information of the quality of peat.

Also the approximate analysis of plant material developed by WAKSMAN and Stevens (12) and modified e.g. by SPRINGer (11) to include the determination of uronic acids has been applied to the study of peat samples. It is however, a too laborious method for the determination of the degree of humification. A more rapid way was proposed by KePpeLeR (3). His "Zersetzungsgrad» was calculated on the basis of the total amount of reducing sugars in the acid hydrolysate of the peat sample, and his "Vertorfungsgrad" was given on the basis of the organic residue of a similar hydrolysis. As a base of comparison an approximate value found for undecomposed Sphagnum moss was employed, and later (4) also the effect of the loss of dry matter during the decomposition was taken into consideration. SEgeberg (10) found on the basis of a material of 31 samples of Sphagnum peat a correlation coefficient of about 0.8 between his "Vertorfungszahl» and KeppeleR's "Vertorfungsgrad».

Some of the aforementioned quantities were determined for eight peat samples. All the analyses except the estimation of von PosT's H-value were performed using air-dry and ground samples. Thus e.g. the "Vertorfungszahl» by SEgEBerg is not 
quite genuine. The colour number does not correspond to the proper "Humifizierungszahl» by Melin and OdÉn. It was determined by extracting the peat sample with $0.5 \mathrm{~N}$ sodium hydroxide in the ratio of 1 to 100 for 18 hours at room temperature and measuring the colour intensity of the filtered and ten-fold diluted extract. The result is reported as the extinction value obtained by a Lumetron colorimeter M 402-E using a monochromatic filter with the transmission peak at $550 \mathrm{~m} \mu$. Table 1 shows the results listed according to the increasing degree of humification by von Post. With some exceptions this was also the order of the increasing V. Z. values by SEGEBERG, but the correlation with the data for maximum water-holding capacity is less evident. The decrease in the cellulose and hemicellulose percentages are well in accordance with the increase in the H-values, whereas the amounts of insoluble residues as well as the "Vertorfungsgrad» by KePPELER change less regularly. The colour number appears to give a rather confused picture, a fact that could be expected, because the light absorption of an extract depends both on the darkness and the amount of organic matter in the sample as well as on its solubility under the conditions in question. In this case, apparently, only an indefinite part of the organic matter was dissolved. The S and CS peats yielded extracts relatively darker than those of the lowmoor peats, an observation made e.g. by SPRINGER (11).

This material is far too scanty to allow any valid comparison of the various characteristics as the indicator of the degree of humification. The samples are also only slightly decomposed. Thus e.g. they all contain measurable amounts of cellulose which is not the case when decomposition is more advanced.

When attempting to determine the degree of humification attention has also been paid to the increase in the nitrogen content of the decomposing material and particularly to the accumulation of acid insoluble or acetyl bromide insoluble nitrogen. The total nitrogen content of a peat sample obviously cannot be of great help, owing to the fact that the original material may have contained a greatly varying amount of nitrogen. As to the residual nitrogen it is difficult to see any advantage in the use of it instead of that of the total residue. The ratio of organic carbon to totalnitrogen has been a fairly common measure for the advancement of decomposition, although it probably is more suitable for the prediction of the possible mobilization or immobilization during the decomposition, provided that the carbon and nitrogen compounds are easily available to microorganisms. As far as the author knows the dependence of the ratio $\mathrm{C} / \mathrm{N}$ on the degree of decomposition in peat samples has not been investigated. Kivinen (5) has reported data for the $\mathrm{C} / \mathrm{N}$ ratio in 88 peat samples for which also the degree of decomposition by von Post is given. This material offers an opportunity to compare the $\mathrm{H}$ - and $\mathrm{C} / \mathrm{N}$-values statistically. The total correlation coefficients obtained were for

$$
\begin{array}{ll}
\text { all the } 88 \text { peat samples } & \mathrm{r}=-0.170 \\
28 \mathrm{Sp} \text { and CSp samples } & \mathrm{r}=-0.437^{*} \\
60 \mathrm{SC} \text {, EuSCp, Cp and BCp samples } & \mathrm{r}=-0.057
\end{array}
$$

Only the correlation coefficient for the Sp and CSp samples is significant at the $5 \%$ level. This total lack of correlation between the $\mathrm{C} / \mathrm{N}$ ratio and $\mathrm{H}$-values in the low- 
moor peats and the poor correlation in the highmoor peats is somewhat surprising. It obviously arises from the fact that the nitrogen content of a peat soil depends more on other factors than on the degree of decomposition.

In the absence of an absolute standard for comparison no valid conclusion can be drawn on the reliability of the different conventional methods for the determination of the degree of humification. This lack of a standard is not only due to an analytical defectiveness but it arises mainly from the fact that differences in the original raw material and in the conditions under which the decomposition takes place lead to products which vary to a great extent. Therefore, it seems to be of no use to employ laborious procedures for the estimation of this more or less vague quantity. Attention must be paid to possibilities offered by rapid and simple methods.

\section{Methods studied}

In the present paper results are reported of an attempt to use two kinds of rapid tests for the determination of the degree of humification in peat samples. In spite of the fact that there are very few theoretical reasons to suppose that the colour intensity of a peat extract should be in a close correlation with the degree of humification, the possibilities to apply a colorimetric method was tested. The other characteristic chosen to indicate the degree of humification was the weight of volume.

The material of these studies consisted of 220 samples of Finnish virgin peat soils. They were taken from various depths and they represented different kinds of peat. The samples were collected by several persons who also estimated the $\mathrm{H}$ degree by von Post for the samples and determined the kind of peat. There are probably subjective errors in these determinations but as such they represent typical material obtained in the practical soil survey. The data are listed in Table 2.

\section{Colorimetric estimation}

The principle in the colorimetric estimation of the degree of humification is to measure the colour intensity of an extract obtained by treating the peat sample with some kind of solution which is able to extract the dark coloured substances more or less quantitatively or at least in an amount closely correlated with the total content of dark brown matter in the sample. A standard preparation is needed, or rather a series of standards that represent various degrees of humification in peat of the same kind as those to be analyzed.

In practice the application of these principles is not quite easy. A quantitative extraction of the dark coloured substances is possible only by several succeeding treatments or by the use of a rather drastic manipulation. The first way is laborious and the second one may lead to marked changes in the colour intensity of the humified substances. It is e.g. known that in alkaline solutions an auto-oxidation of a certain kind of organic matter easily yields dark-coloured compounds. On the other hand, 
Table 2. Colour and volume weight as indicators of the degree of humification in peat samples.

\begin{tabular}{|c|c|c|c|c|c|c|c|c|}
\hline \multicolumn{2}{|c|}{ Sample } & $\begin{array}{l}\text { Depth } \\
\text { dm }\end{array}$ & $\mathrm{pH}$ & $\begin{array}{c}\text { Ash } \\
\%\end{array}$ & $\mathrm{H}$ & Colour & $\begin{array}{c}\text { Volume } \\
\text { weight }\end{array}$ & Vol.w./ \\
\hline \multicolumn{2}{|c|}{1} & 2 & 3 & 4 & 5 & 6 & 7 & 8 \\
\hline & & \multicolumn{7}{|c|}{ Sphagnum peat } \\
\hline & 65 & $0-2$ & 3.7 & 1.4 & $0-1$ & 2 & 0.05 & $1-$ \\
\hline & 94 & $0-2$ & 5.7 & 4.5 & $0-1$ & 3 & 0.05 & $1-$ \\
\hline & 144 & $0-2$ & 5.1 & 1.5 & 1 & 2 & 0.07 & 1 \\
\hline K & 31 & $0-2$ & 4.2 & 5.9 & $0-1$ & 3 & 0.08 & 1 \\
\hline $\mathrm{K}$ & 32 & $4-6$ & 4.4 & 3.9 & $0-1$ & 3 & 0.08 & 1 \\
\hline $\mathrm{K}$ & 21 & $0-2$ & 4.3 & 8.0 & $0-1$ & 3 & 0.09 & $1+$ \\
\hline $\mathrm{K}$ & 34 & $0-2$ & 4.2 & 5.0 & $0-1$ & 9 & 0.09 & $1+$ \\
\hline A & 4 & $3-5$ & 4.7 & 1.0 & 1 & 4 & 0.10 & $1+$ \\
\hline $\mathrm{K}$ & 37 & $0-2$ & 4.5 & 4.9 & 1 & 8 & 0.11 & $11 / 2$ \\
\hline $\mathrm{K}$ & 6 & $1-2$ & 4.5 & 4.2 & $0-1$ & 9 & 0.11 & $11 / 2$ \\
\hline \multirow[t]{2}{*}{ A } & 27 & $0-2$ & 4.5 & 5.2 & $0-1$ & 7 & 0.12 & $11 / 2$ \\
\hline & 36 & $0-2$ & 4.0 & 11.2 & 1 & 9 & 0.12 & $11 / 2$ \\
\hline A & 58 & $1-3$ & 3.8 & 4.2 & $0-1$ & 2 & 0.13 & $2-$ \\
\hline A & 37 & $0-2$ & 4.4 & 10.8 & 1 & 9 & 0.28 & $31 / 2$ \\
\hline A & 31 & $0-2$ & 3.9 & 5.7 & 1 & 9 & 0.29 & $4-$ \\
\hline K & 22 & $2-4$ & 5.0 & 2.8 & $1-2$ & 2 & 0.14 & $2-$ \\
\hline $\mathrm{K}$ & 7 & $2-3$ & 4.6 & 4.5 & $1-2$ & 12 & 0.17 & $2+$ \\
\hline \multirow[t]{2}{*}{ V } & $6 a$ & $1-3$ & 4.9 & 10.6 & $2-3$ & 7 & 0.22 & $3-$ \\
\hline & 66 & $2-5$ & 3.6 & 1.5 & 3 & 3 & 0.09 & $1+$ \\
\hline A & 5 & $5-7$ & 4.9 & 2.1 & 3 & 9 & 0.23 & 3 \\
\hline V & $6 \mathrm{~b}$ & $5-7$ & 5.1 & 7.4 & 3 & 10 & 0.26 & $3+$ \\
\hline A & 1 & $2-3$ & 3.7 & 4.4 & 3 & 27 & 0.29 & $4-$ \\
\hline A & 32 & $3-5$ & 4.0 & 4.0 & 3 & 19 & 0.34 & $4+$ \\
\hline A & 6 & $12-14$ & 4.7 & 2.9 & 4 & 14 & 0.21 & $3-$ \\
\hline V & la & $1-3$ & 4.3 & 7.6 & 4 & 18 & 0.31 & 4 \\
\hline \multirow[t]{2}{*}{$\mathrm{V}$} & $15 a$ & $1-3$ & 4.5 & 8.1 & 4 & 15 & 0.33 & $4+$ \\
\hline & 105 & $0-2$ & 4.4 & 2.2 & $4-5$ & 5 & 0.20 & $21 / 2$ \\
\hline \multirow[t]{2}{*}{$\mathrm{V}$} & lb & $5-7$ & 4.3 & 8.5 & $4-5$ & 25 & 0.33 & $4+$ \\
\hline & 67 & $5-9$ & 3.8 & 2.9 & 5 & 10 & 0.21 & $3-$ \\
\hline A & 2 & $3-4$ & 3.8 & 4.3 & 5 & 32 & 0.49 & $6+$ \\
\hline \multirow[t]{2}{*}{ V } & $15 \mathrm{~b}$ & $5-7$ & 4.4 & 4.9 & 6 & 33 & 0.41 & $5+$ \\
\hline & 68 & $9-11$ & 4.2 & 2.4 & 7 & 19 & 0.32 & 4 \\
\hline A & 3 & $7-10$ & 4.4 & 6.3 & 7 & 22 & 0.38 & $5-$ \\
\hline
\end{tabular}

Carex-Sphagnum peat

$\begin{array}{ccccccccc}\mathrm{V} & 24 \mathrm{a} & 1-3 & 4.5 & 7.9 & 1 & 3 & 0.10 & 1+ \\ & 100 & 0-2 & 5.1 & 7.7 & 1 & 6 & 0.11 & 11 \frac{2}{2} \\ \mathrm{~A} & 6 \mathrm{1} & 1-3 & 4.7 & 9.4 & 1 & 9 & 0.15 & 2- \\ \mathrm{V} & 23 \mathrm{a} & 1-3 & 4.5 & 9.5 & 1-2 & 4 & 0.11 & 11 / 2 \\ \mathrm{~V} & 16 \mathrm{a} & 1-3 & 4.4 & 6.9 & 1-2 & 6 & 0.12 & 11 / 2 \\ & 69 & 0-2 & 4.2 & 3.4 & 2 & 6 & 0.09 & 1+ \\ \mathrm{V} & 2 \mathrm{a} & 1-3 & 4.3 & 5.5 & 2 & 7 & 0.15 & 2 \\ \mathrm{~K} & 38 & 2-4 & 4.6 & 5.0 & 2 & 10 & 0.23 & 3 \\ & 101 & 3-5 & 5.2 & 6.5 & 3 & 10 & 0.14 & 2-\end{array}$




\begin{tabular}{|c|c|c|c|c|c|c|c|c|}
\hline \multicolumn{2}{|c|}{1} & 2 & 3 & 4 & 5 & 6 & 7 & 8 \\
\hline & 107 & $0-2$ & 4.4 & 5.0 & 3 & 14 & 0.16 & 2 \\
\hline V & $24 \mathrm{~b}$ & $5-7$ & 4.8 & 7.5 & 3 & 8 & 0.17 & $2+$ \\
\hline V & $23 \mathrm{~b}$ & $5-7$ & 4.9 & 7.8 & 3 & 8 & 0.19 & $21 / 2$ \\
\hline & 28 & $4-6$ & 4.2 & 2.7 & 3 & 7 & 0.23 & 3 \\
\hline & 37 & $0-2$ & 4.7 & 9.9 & 3 & 16 & 0.33 & $4+$ \\
\hline A & 52 & $1-3$ & 3.8 & 7.8 & 3 & 22 & 0.33 & $4+$ \\
\hline $\mathrm{K}$ & 39 & $4-6$ & 4.6 & 4.3 & $3-4$ & 13 & 0.25 & $3+$ \\
\hline V & $21 \mathrm{a}$ & $1-3$ & 5.0 & 4.9 & $3-4$ & 12 & 0.30 & 4 \\
\hline V & $21 \mathrm{~b}$ & $5-7$ & 5.2 & 9.8 & $3-4$ & 13 & 0.28 & $31 / 2$ \\
\hline A & 28 & $5-7$ & 4.3 & 3.5 & $3-4$ & 19 & 0.30 & 4 \\
\hline & 70 & $3-5$ & 4.4 & 9.7 & 4 & 10 & 0.25 & $3+$ \\
\hline V & $16 \mathrm{~b}$ & $5-7$ & 4.5 & 6.0 & 4 & 20 & 0.29 & $4-$ \\
\hline A & 62 & $5-6$ & 4.3 & 7.6 & 4 & 34 & 0.33 & 4 \\
\hline V & $22 \mathrm{~b}$ & $5-7$ & 5.1 & 4.3 & 4 & 13 & 0.28 & $31 / 2$ \\
\hline V & $2 \mathrm{~b}$ & $5-7$ & 4.2 & 4.6 & 4 & 24 & 0.31 & 4 \\
\hline & 34 & $0-2$ & 4.5 & 12.4 & 4 & 24 & 0.34 & $4+$ \\
\hline V & $22 \mathrm{a}$ & $5-7$ & 4.7 & 13.4 & 4 & 11 & 0.37 & $41 / 2$ \\
\hline & 35 & $0-2$ & 4.5 & 9.4 & 4 & 18 & 0.38 & $5-$ \\
\hline $\mathrm{K}$ & 8 & $3-4$ & 4.6 & 5.5 & $4-5$ & 48 & 0.39 & 5 \\
\hline $\mathrm{K}$ & 33 & $6-8$ & 4.5 & 5.8 & 5 & 10 & 0.25 & $3+$ \\
\hline & 102 & $7-10$ & 5.3 & 4.9 & 5 & 11 & 0.31 & 4 \\
\hline & 29 & $15-20$ & 5.1 & 4.4 & 5 & 11 & 0.33 & $4+$ \\
\hline A & 29 & $8-10$ & 4.1 & 4.0 & 5 & 24 & 0.35 & $41 / 2$ \\
\hline & 106 & $0-2$ & 4.7 & 22.4 & 6 & 6 & 0.26 & $3+$ \\
\hline & 71 & $7-10$ & 4.3 & 6.2 & 6 & 12 & 0.36 & $41 / 2$ \\
\hline K & 42 & $2-4$ & 3.9 & 12.4 & $6-7$ & 32 & 0.39 & 5 \\
\hline A & 46 & $4-6$ & 4.1 & 6.6 & $6-7$ & 41 & 0.49 & $6+$ \\
\hline
\end{tabular}

Sphagnum-Carex peat

$\begin{array}{ccccrcrcc}\mathrm{K} & 28 & 0-3 & 4.5 & 6.5 & 1 & 7 & 0.20 & 21 / 2 \\ \mathrm{~V} & 3 \mathrm{a} & 1-3 & 4.4 & 6.7 & 1 & 6 & 0.14 & 2- \\ \mathrm{A} & 19 & 0-2 & 5.0 & 12.3 & 1-2 & 6 & 0.25 & 3+ \\ \mathrm{A} & 13 & 8-10 & 4.8 & 6.5 & 2 & 7 & 0.20 & 21 / 2 \\ \mathrm{~A} & 12 & 3-5 & 4.8 & 4.0 & 2 & 5 & 0.25 & 3+ \\ \mathrm{A} & 23 & 1-3 & 4.7 & 8.0 & 2 & 12 & 0.38 & 5- \\ \mathrm{V} & 19 \mathrm{a} & 1-3 & 4.7 & 8.1 & 2-3 & 8 & 0.21 & 3- \\ \mathrm{V} & 5 \mathrm{a} & 1-3 & 4.6 & 16.3 & 2-3 & 8 & 0.24 & 3 \\ \mathrm{~K} & 12 & 0-1 & 4.4 & 9.8 & 2-3 & 19 & 0.27 & 31 / 2 \\ \mathrm{~A} & 53 & 1-3 & 4.2 & 11.0 & 3 & 37 & 0.42 & 5+ \\ & 59 & 0-2 & 3.6 & 24.4 & 3 & 7 & 0.23 & 3 \\ \mathrm{~A} & 47 & 2-4 & 4.6 & 9.6 & 3 & 20 & 0.23 & 3 \\ \mathrm{~V} & 3 \mathrm{~b} & 5-7 & 4.3 & 5.1 & 3 & 11 & 0.25 & 3+ \\ \mathrm{V} & 30 & 2-3 & 5.1 & 16.9 & 3 & 8 & 0.26 & 3+ \\ \mathrm{A} & 11 & 0-2 & 4.9 & 16.7 & 3 & 5 & 0.26 & 3+ \\ \mathrm{A} & 16 & 6-8 & 4.5 & 3.7 & 3 & 26 & 0.30 & 4- \\ \mathrm{V} & 5 \mathrm{~b} & 5-7 & 4.9 & 8.5 & 3 & 12 & 0.30 & 4- \\ \mathrm{A} & 35 & 0-4 & 5.5 & 9.3 & 3 & 4 & 0.31 & 4 \\ \mathrm{~V} & 10 \mathrm{~b} & 5-7 & 4.9 & 8.1 & 3-4 & 14 & 0.24 & 3 \\ \mathrm{~V} & 9 \mathrm{~b} & 5-7 & 4.9 & 11.5 & 3-4 & 15 & 0.24 & 3 \\ \mathrm{~V} & 13 \mathrm{a} & 1-3 & 4.4 & 9.7 & 3-4 & 13 & 0.25 & 3+\end{array}$




\begin{tabular}{|c|c|c|c|c|c|c|c|c|}
\hline & 1 & 2 & 3 & 4 & 5 & 6 & 7 & 8 \\
\hline $\mathrm{K}$ & 18 & $0-2$ & 5.5 & 15.4 & $3-4$ & 8 & 0.27 & $3 \frac{1}{2}$ \\
\hline V & $9 \mathrm{a}$ & $1-3$ & 4.9 & 18.9 & $3-4$ & 14 & 0.27 & $31 / 2$ \\
\hline V & $10 \mathrm{a}$ & $1-3$ & 4.7 & 18.4 & $3-4$ & 14 & 0.27 & $31 / 2$ \\
\hline V & $14 \mathrm{a}$ & $1-3$ & 4.5 & 9.2 & $3-4$ & 14 & 0.29 & 4 \\
\hline V & $20 \mathrm{a}$ & $1-3$ & 4.6 & 7.8 & $3-4$ & 15 & 0.33 & $4+$ \\
\hline $\mathrm{K}$ & 24 & $6-8$ & 5.1 & 2.2 & $3-4$ & 8 & 0.35 & $4^{1 / 1} 2$ \\
\hline \multirow[t]{2}{*}{ A } & 49 & $1-3$ & 4.3 & 6.2 & $3-4$ & 20 & 0.34 & $4+$ \\
\hline & 86 & $0-2$ & 4.4 & 4.7 & 4 & 11 & 0.21 & $3-$ \\
\hline V & $11 \mathrm{~b}$ & $5-7$ & 4.8 & 14.4 & 4 & 15 & 0.24 & 3 \\
\hline \multirow[t]{2}{*}{ V } & $8 \mathrm{a}$ & $1-3$ & 4.7 & 15.8 & 4 & 14 & 0.25 & $3+$ \\
\hline & 57 & $0-3$ & 4.8 & 15.5 & 4 & 14 & 0.25 & $3+$ \\
\hline V & 26 & $2-3$ & 5.0 & 5.6 & 4 & 12 & 0.26 & $3+$ \\
\hline \multirow[t]{2}{*}{$\mathrm{V}$} & $11 \mathrm{a}$ & $1-3$ & 4.7 & 22.0 & 4 & 14 & 0.28 & $31 / 2$ \\
\hline & 33 & $0-2$ & 4.7 & 5.1 & 4 & 11 & 0.30 & 4 \\
\hline $\mathrm{A}$ & 20 & $4-6$ & 4.2 & 5.8 & 4 & 19 & 0.30 & 4 \\
\hline $\mathrm{V}$ & $12 \mathrm{a}$ & $1-3$ & 4.4 & 10.8 & 4 & 14 & 0.30 & 4 \\
\hline V & $17 \mathrm{a}$ & $1-3$ & 4.8 & 10.1 & 4 & 19 & 0.30 & 4 \\
\hline V & $17 \mathrm{~b}$ & $5-7$ & 4.9 & 5.8 & 4 & 20 & 0.30 & 4 \\
\hline V & $18 \mathrm{a}$ & $1-3$ & 4.8 & 7.4 & 4 & 18 & 0.32 & 4 \\
\hline V & $18 \mathrm{~b}$ & $5-7$ & 4.9 & 5.7 & 4 & 22 & 0.31 & 4 \\
\hline \multirow[t]{2}{*}{ A } & 15 & $2-4$ & 4.3 & 6.5 & 4 & 29 & 0.34 & $4+$ \\
\hline & 55 & $0-2$ & 5.2 & 25.5 & 4 & 29 & 0.44 & $51 / 2$ \\
\hline V & $8 b$ & $5-7$ & 4.9 & 6.1 & $4-5$ & 14 & 0.25 & $3+$ \\
\hline V & $7 \mathrm{a}$ & $1-3$ & 4.7 & 10.2 & 5 & 21 & 0.29 & 4 \\
\hline \multirow[t]{2}{*}{ A } & 33 & $8-10$ & 4.1 & 3.1 & 5 & 28 & 0.30 & 4 \\
\hline & 60 & $2-10$ & 3.5 & 3.5 & 5 & 14 & 0.32 & 4 \\
\hline $\mathrm{K}$ & 35 & $4-6$ & 4.9 & 6.1 & 5 & 24 & 0.34 & $4+$ \\
\hline V & 31 & $4-6$ & 5.1 & 7.9 & 5 & 9 & 0.35 & $41 / 2$ \\
\hline \multirow[t]{2}{*}{ V } & 27 & $4-6$ & 5.2 & 5.5 & 5 & 13 & 0.37 & 5 \\
\hline & 87 & $3-5$ & 4.5 & 2.6 & $5-6$ & 10 & 0.29 & 4 \\
\hline $\mathrm{K}$ & 23 & $4-6$ & 5.0 & 2.0 & $5-6$ & 9 & 0.32 & 4 \\
\hline V & $19 \mathrm{~b}$ & $5-7$ & 5.1 & 7.4 & $5-6$ & 15 & 0.39 & 5 \\
\hline \multirow[t]{2}{*}{$\mathrm{K}$} & 59 & $0-5$ & 5.3 & 9.6 & $5-6$ & 24 & 0.34 & $4+$ \\
\hline & 58 & $3-7$ & 4.9 & 6.9 & 6 & 19 & 0.25 & $3+$ \\
\hline V & 32 & $9-10$ & 5.2 & 7.4 & 6 & 9 & 0.37 & 5 \\
\hline V & 28 & $9-10$ & 5.3 & 5.0 & 6 & 10 & 0.40 & 5 \\
\hline V & $14 \mathrm{~b}$ & $5-7$ & 4.5 & 5.3 & 6 & 27 & 0.40 & 5 \\
\hline V & $13 \mathrm{~b}$ & $5-7$ & 4.5 & 5.4 & 6 & 32 & 0.40 & 5 \\
\hline V & $12 \mathrm{~b}$ & $5-7$ & 4.4 & 4.1 & 6 & 30 & 0.40 & 5 \\
\hline \multirow[t]{4}{*}{ V } & $20 \mathrm{~b}$ & $5-7$ & 5.5 & 6.9 & 6 & 27 & 0.42 & $5+$ \\
\hline & 56 & $0-2$ & 5.1 & 32.6 & 6 & 48 & 0.43 & $51 / 2$ \\
\hline & 88 & $7-10$ & 5.2 & 4.0 & $6-7$ & 16 & 0.30 & 4 \\
\hline & 61 & $10-13$ & 4.2 & 13.2 & 7 & 35 & 0.40 & 5 \\
\hline \multirow[t]{2}{*}{$\mathrm{V}$} & $7 \mathrm{~b}$ & $5-7$ & 5.0 & 9.1 & 7 & 40 & 0.40 & 5 \\
\hline & 76 & 60 & 4.8 & 11.2 & 9 & 30 & 0.71 & 9 \\
\hline
\end{tabular}

Eutrophic Sphagnum-Carex peat

$\begin{array}{rrrrrrrr}91 & 0-2 & 5.8 & 9.1 & 2 & 2 & 0.18 & 2+ \\ 62 & 0-2 & 4.4 & 18.4 & 3 & 6 & 0.18 & 2+ \\ 40 & 0-2 & 5.6 & 9.8 & 3 & 3 & 0.25 & 3+\end{array}$




\begin{tabular}{|c|c|c|c|c|c|c|c|}
\hline 1 & 2 & 3 & 4 & 5 & 6 & 7 & 8 \\
\hline 95 & $3-5$ & 5.8 & 7.7 & 3 & 20 & 0.36 & $41 / 2$ \\
\hline $\mathrm{K}$ & $3-5$ & 5.4 & 5.3 & $3-4$ & 7 & 0.27 & $31 / 2$ \\
\hline 92 & $3-5$ & 5.9 & 8.2 & 4 & 3 & 0.36 & $4 \frac{1}{2}$ \\
\hline 63 & $2-8$ & 4.7 & 4.9 & 5 & 10 & 0.32 & 4 \\
\hline 96 & $7-10$ & 5.8 & 14.2 & 5 & 75 & 0.41 & $5+$ \\
\hline 93 & $7-10$ & 5.8 & 8.9 & 6 & 4 & 0.41 & $5+$ \\
\hline 117 & $17-20$ & 5.2 & 7.5 & 6 & 25 & 0.41 & $5+$ \\
\hline 118 & $20-23$ & 5.4 & 19.2 & $6-7$ & 59 & 0.47 & 6 \\
\hline 64 & $8-10$ & 4.3 & 5.1 & 7 & 17 & 0.38 & 5 \\
\hline
\end{tabular}

\section{Carex peat}

\begin{tabular}{|c|c|c|c|c|c|c|c|}
\hline 29 & $5-7$ & 4.6 & 5.1 & $1-2$ & 7 & 0.20 & $21 / 2$ \\
\hline 41 & $3-5$ & 4.8 & 5.1 & $2-3$ & 7 & 0.25 & $3+$ \\
\hline 43 & $2-6$ & 4.5 & 5.1 & 3 & 40 & 0.24 & 3 \\
\hline 40 & $0-2$ & 4.7 & 5.5 & 3 & 7 & 0.30 & $4-$ \\
\hline 38 & $3-5$ & 4.5 & 10.0 & 3 & 13 & 0.29 & $4-$ \\
\hline 8 & $0-2$ & 4.7 & 5.0 & 3 & 10 & 0.32 & 4 \\
\hline 24 & $5-6$ & 5.0 & 5.5 & 3 & 15 & 0.34 & $4+$ \\
\hline 25 & $1-3$ & 4.6 & 4.5 & $3-4$ & 26 & 0.21 & $3-$ \\
\hline 26 & $11-14$ & 4.2 & 3.1 & $3-4$ & 37 & 0.20 & $21 / 2$ \\
\hline 14 & $5-7$ & 5.1 & 4.9 & $3-4$ & 17 & 0.26 & $3+$ \\
\hline 13 & $1-3$ & 4.9 & 5.1 & $3-4$ & 18 & 0.28 & $31 / 2$ \\
\hline 38 & $0-2$ & 4.9 & 6.7 & $3-4$ & 13 & 0.36 & $41 / 2$ \\
\hline 27 & $11-14$ & 4.4 & 5.3 & 4 & 48 & 0.23 & $3-$ \\
\hline 109 & $0-2$ & 4.6 & 24.8 & 4 & 5 & 0.24 & 3 \\
\hline 111 & $0-2$ & 4.7 & 3.0 & 4 & 40 & 0.26 & $3+$ \\
\hline 103 & $0-2$ & 4.8 & 7.7 & 4 & 15 & 0.28 & $31 / 2$ \\
\hline 44 & $6-8$ & 4.3 & 6.9 & 4 & 49 & 0.27 & $31 / 2$ \\
\hline 42 & $8-10$ & 4.9 & 4.2 & $4-5$ & 8 & 0.23 & 3 \\
\hline 110 & $0-2$ & 4.6 & 3.6 & $4-5$ & 32 & 0.24 & 3 \\
\hline 17 & $8-10$ & 4.1 & 5.5 & 5 & 31 & 0.31 & $4-$ \\
\hline 36 & $4-6$ & 4.9 & 6.1 & 5 & 10 & 0.34 & $4+$ \\
\hline 41 & $2-6$ & 4.2 & 3.5 & $5-6$ & 47 & 0.28 & $31 / 2$ \\
\hline 30 & $2-5$ & 4.8 & 4.9 & $5-6$ & 26 & 0.34 & $4+$ \\
\hline 104 & $0-2$ & 4.6 & 7.6 & 6 & 32 & 0.29 & $31 / 2$ \\
\hline 20 & $6-8$ & 5.4 & 4.8 & 6 & 25 & 0.30 & $4-$ \\
\hline 131 & $27-30$ & 5.0 & 8.2 & 6 & 11 & 0.35 & $41 / 2$ \\
\hline 25 & $8-9$ & 5.1 & 8.7 & 6 & 32 & 0.37 & $5-$ \\
\hline 138 & $20-23$ & 5.5 & 11.3 & 6 & 38 & 0.43 & $51 / 2$ \\
\hline 4 & $3-6$ & 5.2 & 6.6 & $6-7$ & 20 & 0.35 & $41 / 2$ \\
\hline 21 & $8-10$ & 5.8 & 7.2 & 7 & 12 & 0.37 & $5-$ \\
\hline 137 & $17-20$ & 5.6 & 12.9 & 7 & 34 & 0.39 & 5 \\
\hline 50 & $3-5$ & 4.6 & 5.3 & $7-8$ & 31 & 0.31 & 4 \\
\hline 31 & $3-6$ & 4.9 & 6.3 & $7-8$ & 36 & 0.39 & 5 \\
\hline 19 & $3-5$ & 5.4 & 8.1 & $7-8$ & 16 & 0.46 & $6-$ \\
\hline 30 & $0-3$ & 4.6 & 9.1 & $7-8$ & 55 & 0.54 & $7-$ \\
\hline 32 & $0-5$ & 4.7 & 23.6 & $7-8$ & 84 & 0.69 & 9 - \\
\hline 45 & $12-14$ & 4.9 & 20.6 & 8 & 49 & 0.52 & $61 / 2$ \\
\hline K 60 & $10-14$ & 4.9 & 7.0 & $8-9$ & 15 & 0.53 & 7 - \\
\hline
\end{tabular}




\begin{tabular}{|c|c|c|c|c|c|c|c|c|}
\hline \multicolumn{2}{|c|}{1} & 2 & 3 & 4 & 5 & 6 & 7 & 8 \\
\hline & \multicolumn{8}{|c|}{ Bryales-Carex peat } \\
\hline K & 9 & $0-2$ & 4.9 & 9.9 & $0-1$ & 6 & 0.16 & 2 \\
\hline K & 1 & $0-2$ & 5.5 & 6.3 & 1 & 4 & 0.14 & $2-$ \\
\hline \multirow[t]{13}{*}{$\mathrm{K}$} & 10 & $1-3$ & 5.2 & 15.6 & $1-2$ & 9 & 0.24 & 3 \\
\hline & 74 & $0-2$ & 6.2 & 5.7 & 2 & 6 & 0.20 & $21 / 2$ \\
\hline & 122 & $14-17$ & 4.9 & 8.2 & 2 & 10 & 0.22 & $3-$ \\
\hline & 39 & $0-2$ & 4.7 & 4.2 & $2-3$ & 6 & 0.23 & $3-$ \\
\hline & 119 & $4-7$ & 5.1 & 17.1 & $2-3$ & 7 & 0.30 & $4-$ \\
\hline & 120 & $7-10$ & 5.0 & 6.0 & $2-3$ & 9 & 0.25 & $3+$ \\
\hline & 121 & $10-13$ & 4.8 & 6.8 & $2-3$ & 9 & 0.21 & $21 / 2$ \\
\hline & 129 & $20-23$ & 4.8 & 5.9 & $2-3$ & 14 & 0.27 & $31 / 2$ \\
\hline & 143 & $0-2$ & 4.1 & 3.7 & $2-3$ & 18 & 0.21 & $21 / 2$ \\
\hline & 113 & $4-7$ & 5.6 & 6.7 & 3 & 6 & 0.28 & $31 / 2$ \\
\hline & 126 & $10-13$ & 4.9 & 3.7 & 3 & 8 & 0.25 & $3+$ \\
\hline & 127 & $14-17$ & 4.9 & 4.8 & 3 & 10 & 0.24 & 3 \\
\hline & 128 & $17-20$ & 4.8 & 4.7 & 3 & 12 & 0.25 & $3+$ \\
\hline \multirow[t]{18}{*}{ K } & 2 & $3-5$ & 5.2 & 7.5 & 3 & 13 & 0.28 & $3 \frac{1}{2}$ \\
\hline & 97 & $0-2$ & 3.9 & 4.3 & 3 & 12 & 0.26 & $3+$ \\
\hline & 73 & $0-2$ & 8.0 & 15.1 & 3 & 21 & 0.44 & $51 / 2$ \\
\hline & 125 & $4-7$ & 4.9 & 4.4 & $3-4$ & 8 & 0.30 & 4 \\
\hline & 123 & $17-20$ & 4.9 & 4.4 & $3-4$ & 11 & 0.24 & 3 \\
\hline & 114 & $7-10$ & 5.4 & 7.5 & $3-4$ & 7 & 0.29 & $31 / 2$ \\
\hline & 141 & $0-2$ & 5.6 & 5.1 & 4 & 4 & 0.45 & 6 \\
\hline & 75 & $0-2$ & 5.4 & 7.1 & 4 & 19 & 0.24 & 3 \\
\hline & 130 & $24-27$ & 5.0 & 5.1 & $4-5$ & 11 & 0.36 & $4 \frac{1}{2}$ \\
\hline & 98 & $3-5$ & 4.4 & 3.3 & $4-5$ & 29 & 0.34 & $4+$ \\
\hline & 142 & $0-2$ & 4.8 & 6.6 & 5 & 6 & 0.53 & $7-$ \\
\hline & 115 & $10-13$ & 5.4 & 6.5 & 5 & 8 & 0.37 & 5 \\
\hline & 135 & $10-13$ & 5.8 & 9.8 & $5-6$ & 41 & 0.44 & $51 / 2$ \\
\hline & 116 & $14-17$ & 5.3 & 7.0 & 6 & 14 & 0.39 & 5 \\
\hline & 140 & $0-2$ & 5.1 & 8.9 & 6 & 26 & 0.58 & $7+$ \\
\hline & 99 & $7-10$ & 4.9 & 4.6 & 6 & 34 & 0.45 & $6-$ \\
\hline & 134 & $4-10$ & 5.7 & 15.3 & 6 & 42 & 0.43 & $5^{1 / 2}$ \\
\hline & 136 & $14-17$ & 5.7 & 11.8 & $6-7$ & 37 & 0.42 & $5+$ \\
\hline K & 11 & $5-7$ & 5.0 & 10.6 & 7 & 14 & 0.37 & 5 \\
\hline $\mathrm{K}$ & 3 & $7-9$ & 5.3 & 4.4 & 7 & 18 & 0.34 & $4+$ \\
\hline
\end{tabular}

some decomposition of coloured material may also take place. Thus there are possible sources of error in the fairly drastic extraction of the peat samples in the determination of the proper "Humifizierungszahl».

The defectiveness of the use of one standard, e.g. of the extract of Acidum huminicum by Merck, arises not only from the fact emphasized by Носк (2) that Acidum huminicum represents highmoor peats. The estimation of the degree of humification as a percentage of the colour intensity of a certain preparation probably is too simplified. It would mean that a linear correlation exists between the colour intensity and the degree of decomposition, an assumption which is not proved. The adoption of respective series of preparations for comparison is arbitrary too as long as no absolute standards exist. 
The method adopted in this investigation consisted of an extraction of an 1-g peat sample for 18 hours with $100 \mathrm{ml}$ of $0.025 \mathrm{M}$ sodium pyrophosphate solution at room temperature. The colour intensity was measured from the filtered and fivefold diluted extract with a Lumetron colorimeter M 402-E using the monochromatic light filter which has its transmission peak at $550 \mathrm{~m} \mu$. The results are reported as the extension values multiplied by 100 .

This arbitrary procedure was chosen on the basis of preliminary experiments in which the suitability of various solvents, e.g. of sodium hydroxide, sodium oxalate and sodium fluoride, was studied. It was found that sodium hydroxide extracted a large amount of dark coloured organic substances which, however, did not react as true humic matter in the precipitation and redissolution tests. The purification of the less dark-coloured pyrophosphate, oxalate and fluoride extracts from non-humic matter led only to a slight decrease in their colour intensity. The pH-value of the pyrophosphate extracts was about 9 . Therefore higher amounts of organic matter were extracted by pyrophosphate solution than by oxalate or fluoride solutions.

The results obtained by the pyrophosphate procedure for the peat samples are reported in Table 2 in the column titled "Colour». Generally higher colour numbers are found for the samples that are decomposed to a greater extent according to the von Post's H-values, but marked exceptions also exist. In order to get an idea about the relation of these data they were compared by calculating the total linear correlation coefficients. These were for

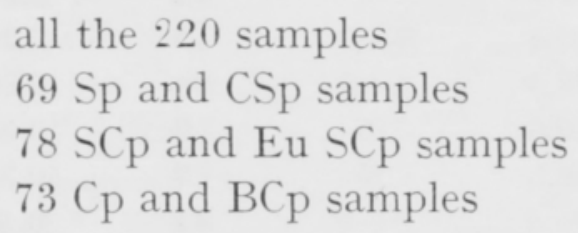

$$
\begin{aligned}
& r=0.588 * * * \\
& r=0.657 * * * \\
& r=0.478 * * * \\
& r=0.580 * * *
\end{aligned}
$$

All these correlations are significant at the $0.1 \%$ level.

As could be expected, no very marked association exists between these quantities. A more thorough examination of the data indicated that in most of the disagreeing cases the colour numbers show a relatively lower degree of humification than the H-values. Since we have no proof of the reliability of the latter, it is possible that in some cases the colour intensity may give a better estimation. Generally, however, it is more probable that some factors have prevented the extraction of the dark coloured matter in these samples or that the formation of these compounds has not advanced at the same speed as the structure of the plant material has disappeared.

It could be supposed that the reaction of the peat may be in connection with the solubility of its organic matter and it thus would exert an effect upon the colour intensity of the extracts. In this material the elimination of the effect of $\mathrm{pH}$ did not improve the correlation between the $\mathrm{H}$-values and colour numbers. The partial correlation coefficient obtained for all the 220 samples was $\mathrm{r}=0.598$.***

An examination of the data does not indicate that a closer correlation of some other kind could be found between these colour numbers and the H-values. Thus, if it is supposed that the H-values by von Post at present yield the best estimation 
of the degree of humification the conclusion must be drawn that the proposed colorimetric method cannot be recommended. It only can be used as a supplement to other estimations of the degree of bumification.

\section{Weight of volume}

Attention has been paid to the association between the volume weight and the degree of humification in peat samples. The volume weight is a quantity commonly determined when peat samples are analyzed. Therefore, if it really could be used as a measure for the degree of humification, it would offer a great help to the peat analyst.

TACKE (12) reports that $1000 \mathrm{~cm}^{3}$ of air-dry and ground undecomposed Sphagnum peat in loose condition weighed $55 \mathrm{~g}$, while the same amounts of younger and older Sphagnum peat weighed $75 \mathrm{~g}$ and $312 \mathrm{~g}$ resp. The corresponding figures for samples treated with a pressure of $10 \mathrm{~kg}$ per $\mathrm{cm}^{2}$ were $106 \mathrm{~g}, 157 \mathrm{~g}$ and $464 \mathrm{~g}$, resp. All these weights were calculated to express the amounts of organic dry-matter.

These observations were further applied to the development of the method by SEGEBERG (10). His "Vertorfungszahl» signifies the difference between the volume weight of the organic dry-matter in the humified sample and that of the undecomposed raw material expressed as a percentage of the former. The volume weight of the ash-free undecomposed highmoor peat is supposed to be 75 , and the specific gravity of the peat ash, a figure needed in the calculation of the volume weight of organic dry matter, is taken to be 1.5. The "Vertorfungszahl» is 0 for undecomposed material and increases with advancing decomposition. The highest "Vertorfungszahl» reported by SEGEBERG was 75 .

SEGEBERG found a rather close negative correlation between the "Vertorfungszahl» and the maximum water-holding capacity of the peat samples. Also the correlation coefficient reported for the "Vertorfungszahl" and KeppeleR's "Vertorfungsgrad" was fairly high. The material used for the analyses, however, consisted of only 31 samples.

The weight of volume of a soil sample depends largely on the method employed. SEGEBERG determined the volume weight using fresh samples bored with a cylinder of known volume. When dry and ground material is used, the data obtained often give only a poor idea of the apparent volume weights under natural conditions. But at the laboratory stage these are generally the estimations with which the analyst has to be contented. In order to get comparable results care must be taken that the determinations are performed using each time the same pressure for the compacting of the material.

For the determination of the volume weights of the present material a simple apparate developed by Mr. Jaakko Kivekäs M. Sci. (Agr.) in this laboratory was use. It is a cylinder in two pieces. The lower part equipped with a fixed bottom and a sliding lid holds $33 \mathrm{~cm}^{3}$. The top part is connected with the lower one and the lid drawn aside. The cylinder is filled with air-dry ground peat and dropped vertically 
from the height of $20 \mathrm{~cm}$ three times in order to bring about an as equal tightness as possible. The top part with superfluous peat is drawn aside and the content of the lower part is weighed. The determinations were performed as four replicates. The variation between these was low, generally far less than five per cent.

The results obtained are reported in Table 2. The ash content of the samples are also listed. Since these percentages appear to be low, in general markedly smaller than $10 \%$, the volume weight calculated for the ash-free material cannot considerably differ from the original value for the whole sample. The first comparisons were, therefore, performed using the original volume weights.

First these data were compared with the $\mathrm{H}$-values by von Post. The total correlation coefficients obtained were the following.
All the 220 peat samples
$\mathrm{r}=0.792 * * *$
69 Sp and CSp samples
$\mathrm{r}=0.813^{* * *}$
$78 \mathrm{SCp}$ and EuSCp samples
$\mathrm{r}=0.728^{* * *}$
$73 \mathrm{Cp}$ and $\mathrm{BCp}$ samples
$\mathrm{r}=0.775^{* * *}$

All these correlation coefficients are significant at the 0.1 per cent level.

These correlation coefficients are higher than those found for the association between the colorimetric data and the $\mathrm{H}$-values. The regression lines of the $\mathrm{H}$-values (y) upon the volume weights $(\mathrm{x})$ were calculated and the following equations were obtained:
All the 220 samples
$\mathrm{y}=12.8 \mathrm{x}-0.17$
$\sigma_{\mathrm{r}}=1.09$
69 Sp and CSp samples
$\mathrm{y}=11.5 \mathrm{x}+0.57$
$\sigma_{\mathrm{r}}=1.04$
78 SCp and EuSCp samples
$\mathrm{y}=12.2 \mathrm{x}-0.21$
$\sigma_{\mathrm{r}}=1.07$
$73 \mathrm{Cp}$ and BCp samples
$\mathrm{y}=12.9 \mathrm{x}+0.26$
$\sigma_{\mathrm{r}}=1.14$

$\sigma_{\mathrm{r}}$ is the residual variance about the regression line, measured in units of $\mathrm{y}$. Thus the $95 \%$ confidence limits on either side of these regression lines would be on the distance of about two H-units from it. Consequently, the accuracy of the prediction of $\mathrm{H}$-values from a known value of volume weight is not very high .

It could be supposed that the ash content of the sample would have some effect upon its volume weight. This, of course, is the fact when the sample contains mineral matter. These samples, however, were taken from virgin peat soils, and as far as known their ash consisted only of peat ash. In this material the correlation coefficients between the volume weight and the ash content were very low or nonsignificant:

for all the 220 samples

$69 \mathrm{Sp}$ and CSp samples

78 SCp and EuSCp samples

$73 \mathrm{Cp}$ and $\mathrm{BCp}$ samples

$$
\begin{aligned}
& r=0.247^{* *} \\
& r=0.271^{*} \\
& r=0.011 \\
& r=0.342^{* *}
\end{aligned}
$$


The partial correlation coefficients between the $\mathrm{H}$-values and the volume weights after the elimination of the effect of ash content were almost equal to the corresponding total correlation coefficients or

$$
\begin{array}{cl}
\text { for all the } 220 \text { samples } & \mathrm{r}=0.790^{* * *} \\
69 \mathrm{Sp} \text { and CSp samples } & \mathrm{r}=0.805^{* * *} \\
78 \mathrm{SCp} \text { and EuSCp samples } & \mathrm{r}=0.740^{* * *} \\
73 \mathrm{Cp} \text { and } \mathrm{BCp} \text { samples } & \mathrm{r}=0.773^{* * *}
\end{array}
$$

Consequently, the ash content of the virgin peat samples of the material studied has no effect upon the association of the volume weight with the corresponding $\mathrm{H}$-values. The calculation of the volume weight of organic dry matter would give a no better estimation of the degree of humification of virgin peat samples.

It is of interest to find out whether the correlation between the colour number and the volume weight is higher or lower than the corresponding figure for the $\mathrm{H}$ values and the volume weights. The results obtained were the following:

$\begin{array}{ll}\text { All the } 220 \text { samples } & \mathrm{r}=0.597^{* * *} \\ 69 \text { Sp and CSp samples } & \mathrm{r}=0.800^{* * *} \\ 78 \text { SCp and EuSCp samples } & \mathrm{r}=0.568^{* * *} \\ 73 \mathrm{Cp} \text { and BCp samples } & \mathrm{r}=0.288^{*}\end{array}$

The association between the colour number and the volume weight appears, according to all the samples, to be as close as the correlation of the colour number with the $\mathrm{H}$-values. As to the various peat groups large differences are to be found. The colour numbers of the Sp and CSp samples show a far closer correlation with the volume weights than they do with the $\mathrm{H}$-values $(\mathrm{r}=0.657)$. The opposite is true with the $\mathrm{BC}$ and $\mathrm{Cp}$ group. Thus it seems not to be impossible to employ the colour number when the degree of humification is to be estimated for peat samples consisting mainly of Sphagnum residues.

In the lack of anything better it seems to be justified to use the volume weight of virgin peat samples to characterize their degree of humification. In order to get a convenient scale a simple grading may be adopted. Since it was found that the volume weight of air dry and ground samples of the surface vegetation was, on the average, about 0.08 when determined by the method used in these analyses, this figure was taken as a basic unit. The new degree of humification is the volume weight divided by 0.08 . If another method for the determination of the volume weight is employed, the basic unit is the corresponding value obtained for the undecomposed surface vegetation.

The figures calculated for the new degree of humification are listed in Table 2 under the title Vol.w. 0.08 . Generally, they seem to deviate fairly little from the corresponding $\mathrm{H}$-values by von Post. In the lower part of the scales the new degree of humification appears to represent somewhat higher values than the figures of von Post, in the higher part the opposite is true. When the average volume weights for 
the various H-grades were calculated for this material the following results were obtained.

$\begin{array}{cccc}\mathrm{H} & \text { Number of samples } & \text { Average } w / \mathrm{v} & \begin{array}{c}\text { New degree of } \\ \text { humification }\end{array} \\ 1 & 25 & 0.12 & 11 / 2 \\ 2 & 17 & 0.22 & 3- \\ 3 & 58 & 0.26 & 3+ \\ 4 & 48 & 0.30 & 4- \\ 5 & 25 & 0.35 & 41 / 2 \\ 6 & 27 & 0.39 & 5- \\ 7 & 15 & 0.39 & 5- \\ 8 & 4 & 0.57 & 7+ \\ 9 & 1 & 0.71 & 9-\end{array}$

The most striking disagreement between these gradings according to this material is to be found in the $\mathrm{H}_{7}$-class, where the average volume weight and therefore also the new degree of humification are exactly the same as in the $\mathrm{H}_{6}$-class. If no attention is paid to the following classes with their markedly higher volume weights, but with only very few samples, it could be supposed that by advancing humification volume weights would increase the less the more decomposed the samples are. This would mean an association between the $\mathrm{H}$-values and the volume weights presented best by a parabel. However, this material does not yield a better correlation of that kind between these quantities. The total correlation coefficient calculated between the logarithms of the $\mathrm{H}$-values and volume weights was $\mathrm{r}=0.818$, not significantly higher than the corresponding figure for the original numbers.

If the differences between the degrees of humification of the individual samples are examined, the greatest differences can be found among the samples taken from the deeper layers. For these samples the volume weight gives a lower degree of humification than do the $\mathrm{H}$-values. It is quite possible that in some of these cases the objectiveness of the direct examination has been disturbed by the knowledge that the sample in question originated from old deposits. But the truth may also be that the estimation of the degree of humification on the basis of the volume weight is not a satisfactory method for the more humified samples.

\section{Discussion}

The object of the present investigation which was to find some rapid and simple way to estimate the degree of humification in peat sampels, is not a problem which could be easily dissolved. An examination of the theoretical possibilities clearly reveals this fact. At present, only very approximate methods can be developed.

As emphasized above the most difficult problem is the lack of absolute standards for the degrees of humification. The direct examination and the use of the grading by von Post is not satisfactory enough, because it is exposed to a marked subjective variation. In the field work, however, it probably is the only rapid and convenient way to estimate the degree of humification. 
The poor correlation of the colorimetric data obtained by the method developed with the $\mathrm{H}$-values estimated in the field do not recommend the use of this kind of analyses for the determination of the degree of humification. As a supplement method, particularly in the study of peat samples containing mostly Sphagnum residues, it, however, seems to be of some value.

The use of the volume weight as a basis of the estimation of the degree of humification also has rather poor theoretical grounds. It has been found that generally the volume weight increases with advancing decomposition, but the reasons for this are not quite clear. An increase in the ash content obviously plays only a very small part in this case. The reason must be found in the more or less indefinite changes in the structure of the peat material. In samples under natural conditions this change probably is far more distinct than in air-dry and ground material. In the latter the disappearance of the cell structure has, of course, its effect, but attention must be paid also to the different shrinking during the drying due to the different colloidal properties of the undecomposed and decomposed material.

Because the material available in the present studies contained only a small amount of very well humified samples, no valid conclusion can be drawn on the availability of the proposed method to show a distinct difference between various degrees of humification when that kind of samples are in question. In virgin peat soils of Finland very well humified samples are rarely found, and even then generally only in the lower layers of peat deposits.

The use of volume weight is naturally an arbitrary method but in lack of anything better it can be employed. Particular attention must be paid to the fact that when comparable results are needed the determinations of the volume weights must be carried out under strictly similar conditions. Instead of the humification scale adopted here any other can be applied. Although this material did not significantly corroborate the supposition that the increase in the volume weight would be the lower the more advanced stage of humification is in question, a scale based on this kind of grading could possibly be more available than the simple linear scale adopted in this work, particularly, when lowmoor peats are in question.

\section{Sum $m$ ary}

The theoretical difficulties in the determination of the degree of humification in peat samples is discussed and the possibilities offered by some conventional methods are examined.

A colorimetric method based on the extraction of air dry and ground peat samples with $0.025 \mathrm{M}$ sodium pyrophosphate solution was compared with the direct examination of fresh samples by the method of von Post. A significant but not very high correlation was found between the data given by these two methods. It was supposed that colorimetric prodecures of this kind can only be used as supplements of other methods. 
A rather close correlation was found between the volume weights of air-dry and ground samples and the corresponding values determined by the method of von Post. Because of this result and in view of the fact that the volume weight is a quantity generally determined always when peat analyses are performed, it would offer a convenient basis for the estimation of the degree of humification.

Since the material studied contained only some very well humified samples the applicability of the volume weight to the determination of the degree of decomposition of this kind of samples was not proved. A simple scale of humification based on the volume weight was adopted. Its basic unit was 0.08 or the volume weight obtained for the undecomposed plant material collected from various kinds of peat land. It depends, of course, on the method employed for the determination of the volume weight.

The elimination of the effect of the ash content of these virgin peat samples did not significantly improve the correlation of the $\mathrm{H}$-values and the volume weights.

The material of this investigation consisted of 220 samples collected from various depths of virgin peat soils.

\section{Acknowledgement}

The author wishes to express her gratitude to Miss Sylvi Soini M. Sci. (Agr.) and Mr. Jaakko Kivekäs M. Sci. (Agr.) for their help in collecting samples and performing some of the analyses.

\section{REFERENCES}

(1) Arntz, E. 1909. Studien über Tonbestimmung im Boden. Landw. Versuchsstat. 70: 269-306.

(2) Носк, А. 1938. Beziehungen zwischen Konzentration und Farbwerten von Huminsäurelösungen. Bodenk. u. Pflanzenern. 7: 99-117.

(3) Keppeler, G. 1920. Bestimmung des Vertorfungsgrades von Moor- und Torfproben. J. Landw. 68: $43-73$.

(4) —- 1932. Zur Kenntnis des Vertorfungsvorganges. Angew. Chem. 45: 473-476.

(5) Kivinen, E. 1933. Suokasvien ja niiden kasvualustan kasvinravintoainesuhteista. (Referat: Untersuchungen über den Gehalt an Pflanzennährstoffen in Moorpflanzen und an ihren Standorten.) Acta agr. fenn. 27. Helsinki.

(6) LaAtsch, W. 1948. Untersuchungen über die Bildung und Anreicherung von Humusstoffen. Beitr. Agrarwiss. 3: 3-23.

(7) Melin, E. \& OdÉn, S. 1916. Kolorimetrische Untersuchungen über Humus und Humifizierung. Sveriges Geol. Unders. C 278. Stockholm.

(8) Mrtchell, G. F. 1954. The comprehensive examination of peat. Intern. Peat Symp. Dublin 1954, B 2: 7 .

(9) Post, L. von 1924. Das genetische System der organogenen Bildungen Schwedens. Com. Intern. Pédol. IV Comm. 22: 287-304.

(10) Segeberg, H. 1952. Ein Verfahren zur Beurteilung des Vertorfungsgrades von Hochmoortorfen mit Hilfe des Volumengewichtes. Landw. Forsch. 3: 144-155. 
(11) Springer, U. 1940. Humifizierung und Zersetzung und ihre Bestimmung in Torfen, Stallmisten und anderen organischen Bildungen. Bodenk. u. Pflanzenern. 18: 129-167.

(12) TAcke, B. 1930. Die Humusböden der gemässigten Breiten. Handbuch der Bodenlehre IV: 124 184. Berlin.

(13) Waksman, S. A. \& Stevens, K. R. 1930. A system of proximate chemical analysis of plant materials. Ind. Eng. Chem., Anal.. Ed. 2: 167-173.

\title{
SELOSTUS:
}

\section{TURPEEN MAATUMISASTEEN MÄÄRITTÄMISESTÄ}

\author{
Armi Kaila
}

Yliopiston maanviljelyskemian laitos, Helsinki

Tutkimuksessa tarkastellaan turpeen maatumisasteen määrittämiseen liittyviä teoreettisia ja käytännöllisiä vaikeuksia. Absoluuttisten vertailuperusteiden puute tekee mahdottomaksi saada varmuutta eri sovinnaisten menetelmien luotettavuudesta.

Tutkimusaineistona oli 220 luonnontilaisilta soilta otettua turvenäytettä. Selvitettiin yksinkertaisen kolorimetrisen menetelmän antamien tulosten sekä näytteiden tilavuuspainojen suhdetta tavanmukaisesti määritettyyn von Postin maatumisasteeseen. Värilukujen ja $\mathrm{H}$-arvojen välinen vuorosuhde oli merkitsevä, joskin heikohko; sen sijaan tilavuuspainon ja $\mathrm{H}$-arvon positiivinen vuorosuhde oli siksi voimakas, että tilavuuspainon voidaan katsoa ainakin suurin piirtein kuvastavan maatumisastetta. Näytteiden tuhkan pitoisuuden eliminointi ei vaikuttanut tilavuuspainon ja $\mathrm{H}$-arvon väliseen korrelaatioon. Koska tutkitussa aineistossa oli erittäin hyvin maatuneita turpeita niukasti, ei tilavuuspainoon perustuvan maatumisasteen arvioinnin soveltuvuutta tällaisten näytteiden tutkimiseen voitu selvittää.

Tulosten perusteella päädytään siihen, että paremman puutteessa voidaan tilavuuspainoa käyttää ilmoittamaan kuivien ja hienonnettujen turvenäytteiden maatumisastetta, mikäli näytteissä ei ole kivennäisainesta. Menetelmän etuna on sen yksinkertaisuus ja se seikka, että tilavuuspaino tavallisesti muutenkin määritetään turveanalyysien yhteydessä. Tulosten vertailun helpottamiseksi ehdotettiin käytettäväksi arvoa tilavuuspaino jaettuna 0.08:lla, joka saatiin hajaantumattoman pintakasvillisuuden keskimääräiseksi tilavuuspainoksi. Näin ilmoitetut tulokset vastaavat suurin piirtein von Postin maatumisasteita. 